\title{
Recognizable Investigation on Home Visiting Physical Therapy of Physical Therapist in Gwangju and Jeonnam
}

\author{
Seong-Hun $\mathrm{Yu} \cdot$ Seung-Rae $\mathrm{Kim}^{1} \cdot$ Sung-Hyoun $\mathrm{Cho}^{2} \cdot$ Tae-Hwa Seo $^{3} \cdot$ Young-Sung Moon ${ }^{1} \cdot$ \\ Myeong-Hui $\mathrm{Kim}^{4} \cdot$ Hyun-Jin $\mathrm{Kim}^{5 \dagger}$ \\ Gwangju Trauma Center \\ ${ }^{1}$ Gwangju Donggu Public Health Center \\ ${ }^{2}$ Department of Physical Therapy, Nambu University \\ ${ }^{3}$ Sangmu Healing Rehabilitation Convalescent Hospital \\ ${ }^{4}$ Chosun University Dental Hospital \\ ${ }^{5}$ Department of Physical Therapy, Hanlyo University
}

Received: May 5, 2016 / Revised: May 20, 2016 / Accepted: June 15, 2016

(c) 2016 J Korean Soc Phys Med

\section{| Abstract |}

PURPOSE: This study seeks to examine the awareness and need of home visiting physical therapy among physical therapist in Gwangju and Jeonnam province.

METHODS: Survey was conducted during the continuing education on physical therapist held in Gwangju in 2015 with 297 participants. The survey questionnaire consisted of 12 questions on the characteristics of physical therapists, 8 questions regarding the awareness of home visiting physical therapy, and 7 questions on need.

RESULTS: Significant differences were found regarding service period, according to categories of the inconvenience associated with commuting to/from hospitals, long-term treatment for patients, high quality treatment with intensive care, treatment at a time of patients' choosing. The category

†Corresponding Author : gini51@naver.com

This is an Open Access article distributed under the terms of the Creative Commons Attribution Non-Commercial License (http://creativecommons.org/licenses/by-nc/3.0) which permits unrestricted non-commercial use, distribution, and reproduction in any medium, provided the original work is properly cited. of hospital fees reduced due to early discharge from the hospital also saw significant differences, depending on respondents' marital status, dependent family, and service period. Providing treatment without causing discomfort to patients in front of others also saw significant differences according to physical therapist career period. Home visiting physical therapy is easier for family members to take care of patients also saw significant differences according to education and physical therapist career period.

CONCLUSION: Survey indicates that physical therapist province feel the need for home visiting physical therapy in many respects. However, since as many as half of the surveyed physical therapists have only heard of home visiting physical therapy or are have no awareness of the this form of treatment, it appears that there is a need to raise awareness of home visiting physical therapy among physical therapist and introduce appropriate policies.

Key Words: Awareness, Home visiting physical therapy, Physical therapist 


\section{Introduction}

Rapid economic growth, developments in cutting-edge technology, and improvements in medical science have affected health and the lifespan of human beings. As a result, qualitative improvements in life have taken place while inducing an aging society as a new social problem. As the ratio of aged people increases in the entire population of Korea, there is an increase in elderly patients with chronic diseases which requires more demand for rehabilitation treatment for recovery and maintenance of their body functions (Yoon, 2009). Such cases are frequently lead to long-term hospital treatment, causing several problems in terms of physical, economic, and psychological aspects both for the patients and their family members (An, 2009; Bae, 2003). In this context, the necessity of home visiting physical therapy has been consistently presented in order to resolve socially-increasing problems of the vulnerable in medical care and as an alternative from of long-term management of diseases and health promotion (Ahn and Yu, 2012; Hwang et al., 2003).

When the system of home visiting physical therapy is settled, bed turnover rate and effective utilization of professional manpower and facilities can be improved by preventing hospital centralization due to long-term hospitalization, providing effective physical therapy services, preventing social problems by effective patient management in home, inhibiting unnecessary hospitalization, and inducing early discharge of long-term patients who do not require hospitalization. Minimizing unnecessary use of hospitals can have several advantages such as reduction in time and medical cost, fast recovery of patients, and satisfaction of desires on treatment of the disabled at home have been sufficiently presented in other researches (Bae, 2003; Hwang et al., 2003). Home visiting physical therapy is also effective on improving quality of life by enhancing motor skills, enhancing the ability of performing activities in daily life, providing increases in movement and balance ability, and maintaining the active joint working range to contribute to maintaining and enhancing body functions of the old people (Gill et al., 2002; Mangione et al., 2008). Thus, it may be essential to provide systematic home visiting therapy programs for improving motor skills such as endurance and balance ability (Duncan et al., 2003).

Because home visiting physical therapy has sufficient usefulness in increasing the quality of life of patients and in terms of practicality such as reducing in medical coast, it has the potential to become a useful and effective system for satisfaction and necessity of patients (Kim, 2013; Kwon et al., 2014). However, implementation of the system has difficulties due to institutional incompletion. Local home visiting physical therapy which is now being implemented has increased its roles, and it is required to perform several roles or functions as well as its own tasks. In addition, duplication or ambiguous separation of jobs that can be shown when nurses, nutritionist, care helpers, and social works perform jobs as a team with physical therapists, which sometimes leads to ineffective job performance (Ahn and $\mathrm{Yu}, 2012$ ). Such ineffectiveness interrupts professional implementation of home visiting physical therapy and causes difficulty in active performance because of the uncertain matter of responsibility. It reduces the quality of treatment as appropriate services cannot be provided for the recipients, negatively affecting overall effectiveness of local integrated health promotion programs. Further research may be needed to consistently study home visiting physical therapy because the roles and demands of physical therapists are expected to increase in future rehabilitation projects in local communities.

The purpose of this study, in this context, was to investigate the perception and need of home visiting physical therapy among physical therapists in Gwangju and Jeollanam-do. 


\section{Methods}

\section{Subjects and methods}

The subjects of this study were physical therapists, who participated in supplementary education of physical therapy in Gwangju in 12, April, 2015. We explained the purpose of this study to them, and a total of 297 people agreed to participate in the survey of this study. Survey was collected after the supplementary education.

\section{Experimental methods}

We used a questionnaire including twelve items of general characteristics of physical therapist, eight items of their level of awareness of home visiting physical therapy, and seven items regarding the need for home visiting physical therapy. This survey was made on the basis of the literature; physical therapy processor and doctor's degree decided appropriate question number and details (Ahn and Yu, 2012; Han et al., Kim and Lee, 2014; Lee et al., 2014). The subjects were asked to fill in the questionnaires without writing their names. The validity and reliability of the survey results showed that high.

\section{Data analysis}

The SPSS 18.0 program was used to analyze the results of the survey. A frequency analysis was used to analyze the general characteristics of the subjects, level of awareness, and the need of home visiting physical therapy. An independent t-test was used to identify the need for home visiting physical therapy based on sex, marital status, dependent family, position, and site of the general characteristics of the subjects. ANOVA for each group was performed to verify satisfaction of supplementary education based on age, education, monthly pay, physical therapist career period, working week, service period, and 1 day number of patient care. For the level of statistical significance was set at $a=.05$.

\section{Results}

1. General characteristics of physical therapist

Out of the subjects who participated in the survey, $67.30 \%$ were female and the age group of $30 \sim 39$ years old occupied the most shares, or $42.40 \%$. Those who graduated from college occupied $56.90 \%$ as the largest group, those who were unmarried were $61.60 \%$, and those who had no dependents were $57.60 \%$. The highest percentage of each item was as follows: $43.80 \%$ earned monthly paid $1,510,000 \sim 2,000,000$ won per month; $38.00 \%$ had a physical therapist career period of less than five years; $72.10 \%$ were physical therapists; $70.00 \%$ worked 41 49 hours per week; $76.10 \%$ lived in Gwangju; $73.40 \%$ had a service period of less than five years; and $24.90 \%$ cared for $31 \sim 40$ patients per day (Table 1).

Table 1. General characteristics of physical therapist

\begin{tabular}{|c|c|c|c|}
\hline \multicolumn{2}{|c|}{ characteristics } & $\mathrm{N}$ & $\%$ \\
\hline \multirow[t]{2}{*}{ Sex } & Male & 97.00 & 32.70 \\
\hline & Female & 200.00 & 67.30 \\
\hline \multirow[t]{3}{*}{ Age } & $20 \sim 29$ years old & 116.00 & 39.10 \\
\hline & $30 \sim 39$ years old & 126.00 & 42.40 \\
\hline & 40 years old $<$ & 55.00 & 18.50 \\
\hline \multirow[t]{3}{*}{ Education } & College graduate & 177.00 & 59.60 \\
\hline & University graduate & 97.00 & 32.70 \\
\hline & A postgraduate school & 23.00 & 7.70 \\
\hline \multirow[t]{2}{*}{ Marital status } & Unmarried & 183.00 & 61.60 \\
\hline & Married & 114.00 & 38.40 \\
\hline \multirow{2}{*}{$\begin{array}{l}\text { Dependent } \\
\text { family }\end{array}$} & No & 171.00 & 57.60 \\
\hline & Yes & 126.00 & 42.40 \\
\hline \multirow[t]{5}{*}{ Monthly pay } & $<1,500,000$ & 48.00 & 16.20 \\
\hline & $1,510,000 \sim 2,000,000$ & 130.00 & 43.80 \\
\hline & $2,010,000 \sim 2,500,000$ & 57.00 & 19.20 \\
\hline & $2,510,000 \sim 3,000,000$ & 42.00 & 14.10 \\
\hline & $3,010,000<$ & 20.00 & 6.70 \\
\hline \multirow{3}{*}{$\begin{array}{l}\text { Physical } \\
\text { therapist career } \\
\text { period }\end{array}$} & $<5$ years old & 113.00 & 38.00 \\
\hline & $6 \sim 9$ years old & 106.00 & 35.70 \\
\hline & 10 years old $<$ & 78.00 & 26.30 \\
\hline
\end{tabular}




\begin{tabular}{|c|c|c|c|}
\hline \multirow[t]{3}{*}{ Position } & $\begin{array}{l}\text { Manager of physical } \\
\text { therapy }\end{array}$ & 76.00 & 25.60 \\
\hline & Physical therapy & 214.00 & 72.10 \\
\hline & And so on & 7.00 & 2.40 \\
\hline \multirow[t]{3}{*}{ Working week } & $<40$ times & 66.00 & 22.20 \\
\hline & $41 \sim 49$ times & 208.00 & 70.00 \\
\hline & 50 times $<$ & 23.00 & 7.70 \\
\hline \multirow[t]{2}{*}{ Site } & Gwangju & 226.00 & 76.10 \\
\hline & Jeonnam & 71.00 & 23.90 \\
\hline \multirow[t]{3}{*}{ Service period } & $<5$ years old & 218.00 & 73.40 \\
\hline & $6 \sim 9$ years old & 58.00 & 19.50 \\
\hline & 10 years old $<$ & 21.00 & 7.10 \\
\hline \multirow{6}{*}{$\begin{array}{l}1 \text { day number } \\
\text { of patient care }\end{array}$} & $<10$ peoples & 25.00 & 8.40 \\
\hline & $11 \sim 20$ peoples & 67.00 & 22.60 \\
\hline & 21 30 peoples & 71.00 & 23.90 \\
\hline & $31 \sim 40$ peoples & 74.00 & 24.90 \\
\hline & 41 peoples $<$ & 47.00 & 15.80 \\
\hline & And so on & 13.00 & 4.40 \\
\hline
\end{tabular}

2. Level of awareness of home visiting physical therapy

For the question "Are you aware of home visiting physical therapy?", $43.40 \%$ of the participants answered "I know what it is to some extent". For the question "Do you think home visiting physical therapy is necessary?", $50.20 \%$ said "Necessary". $42.00 \%$ of the participants chose "Stroke" for the question "For what kind of illness do you think it is particularly needed?". $46.40 \%$ of them chose "Public institution" for the question "Which organization would you like to carry out home visiting physical therapy?". For the question "What days are good for home visiting physical therapy?", $62.60 \%$ (the highest) answered they "decided in consultation with patients". When asked "How many hours do you think are appropriate for home visiting physical therapy?", $66.60 \%$ (the highest) answered "One hour". For the question "What kind of treatment would you like when receiving home visiting physical therapy?", $48.80 \%$ (the highest) chose "Activities of daily living treatment". For the question "What level of qualifications do you think is appropriate for physical therapist to provide home visiting physical therapy?", $41.40 \%$ (the highest) of them said "At least 5 years of clinical experience" (Table 2).

\section{Need for home visiting physical therapy}

When asked "Do you think home visiting physical therapy helps reduce the inconvenience associated with commuting to/from hospital?", "Do you think home visiting physical therapy is appropriate for patients who need long-term treatment?", "Do you think early discharge from hospital can help reduce hospital fees?", "Do you think that with home visiting physical therapy, intensive care is possible and effective treatment can be provided?", "Do you think with that home visiting physical therapy can provide treatment without causing discomfort to patients in front of others?", "Do you think that with home visiting physical therapy, it is easier for family members to take care of patients?", and "Do you think that home visiting physical therapy can be provided at a time patients choose by way of advance appointment?", 62.30\%, 61.30\%, $47.80 \%, 54.20 \%, 60.60 \%, 54.20 \%$, and $54.90 \%$ (the highest) of the participants chose the answer "Satisfied" (Table 3).

\section{Need for home visiting physical therapy in} terms of physical therapist characteristics

For the question "Do you think early discharge from hospital can help reduce hospital fees?", there were significant differences according to marital status, dependent family, and service period. For the question "Do you think that with home visiting physical therapy, it is easier for family members to take care of patients?", significant differences were found according to education and physical therapist career period. For the question "Do you think with that home visiting physical therapy can provide treatment without causing discomfort to patients in front of others?", there was significant change in physical therapist career period. As for service period, in particular, 
Table 2. Level of awareness of home visiting physical therapy

\begin{tabular}{|c|c|c|c|}
\hline characteristics & & $\mathrm{N}$ & $\%$ \\
\hline \multirow[t]{4}{*}{ Are you aware of home visiting physical therapy? } & Not at all & 32.00 & 10.80 \\
\hline & I have heard of it & 116.00 & 39.10 \\
\hline & I know what it is to some extent & 129.00 & 43.40 \\
\hline & I know it well enough & 20.00 & 6.70 \\
\hline \multirow[t]{5}{*}{ Do you think home visiting physical therapy is necessary? } & Very unnecessary & 3.00 & 1.00 \\
\hline & Unnecessary & 16.00 & 5.40 \\
\hline & Natural & 95.00 & 32.00 \\
\hline & Necessary & 149.00 & 50.20 \\
\hline & Very necessary & 34.00 & 11.40 \\
\hline \multirow[t]{8}{*}{ For what kind of illness do you think it is particularly needed? } & Dementia & 16.00 & 5.40 \\
\hline & Stroke & 125.00 & 42.00 \\
\hline & Cerebral palsy & 35.00 & 11.80 \\
\hline & Parkinson's disease & 2.00 & .70 \\
\hline & Arthritis & 8.00 & 2.70 \\
\hline & Spinal cord injury & 32.00 & 10.80 \\
\hline & Pain & 8.00 & 2.70 \\
\hline & And so on & 71.00 & 23.90 \\
\hline \multirow{5}{*}{$\begin{array}{l}\text { Which organization would you like to carry out home visiting } \\
\text { physical therapy? }\end{array}$} & Public institution & 138.00 & 46.40 \\
\hline & General hospital & 8.00 & 2.70 \\
\hline & Social organization & 15.00 & 5.10 \\
\hline & Physical therapy association & 100.00 & 33.70 \\
\hline & And so on & 36.00 & 12.10 \\
\hline \multirow[t]{5}{*}{ What days are good for home visiting physical therapy? } & Days designated by doctors & 6.00 & 2.00 \\
\hline & On a regular basis & 84.00 & 28.30 \\
\hline & When patients desire & 16.00 & 5.40 \\
\hline & Decided in consultation with patients & 186.00 & 62.60 \\
\hline & And so on & 5.00 & 1.70 \\
\hline \multirow{5}{*}{$\begin{array}{l}\text { How many hours do you think are appropriate for home } \\
\text { visiting physical therapy? }\end{array}$} & 30 minute & 67.00 & 22.60 \\
\hline & 1hours & 198.00 & 66.60 \\
\hline & 1hours 30minute & 22.00 & 7.40 \\
\hline & 2hours & 5.00 & 1.70 \\
\hline & And so on & 5.00 & 1.70 \\
\hline \multirow{5}{*}{$\begin{array}{l}\text { What kind of treatment would you like when receiving home } \\
\text { visiting physical therapy? }\end{array}$} & Manual treatment & 32.00 & 10.80 \\
\hline & Therapeutic treatment & 86.00 & 29.00 \\
\hline & Activities of daily living treatment & 145.00 & 48.80 \\
\hline & Pain treatment & 6.00 & 2.00 \\
\hline & And so on & 28.00 & 9.40 \\
\hline \multirow{5}{*}{$\begin{array}{l}\text { What level of qualifications do you think is appropriate for } \\
\text { physical therapist to provide home visiting physical therapy? }\end{array}$} & At least 3 years of clinical experience & 112.00 & 37.70 \\
\hline & At least 5 years of clinical experience & 123.00 & 41.40 \\
\hline & At least 10 years of clinical experience & 32.00 & 10.80 \\
\hline & Any chartered physical therapist & 28.00 & 9.40 \\
\hline & And so on & 2.00 & .70 \\
\hline
\end{tabular}

significant changes were shown for the questions "Do you think home visiting physical therapy helps reduce the inconvenience associated with commuting to/from hospital?", "Do you think home visiting physical therapy is appropriate for patients who need long-term treatment?", "Do you think that with home visiting physical therapy, intensive care is possible and effective treatment can be provided?", and "Do you think that home visiting physical 
Table 3. Need for home visiting physical therapy

\begin{tabular}{|c|c|c|c|}
\hline characteristics & & $\mathrm{N}$ & $\%$ \\
\hline \multirow{5}{*}{$\begin{array}{l}\text { Do you think home visiting physical therapy helps reduce the inconvenience } \\
\text { associated with commuting to/from hospital? }\end{array}$} & Very dissatisfied & 2.00 & .70 \\
\hline & Dissatisfied & 8.00 & 2.70 \\
\hline & Neutral & 80.00 & 26.90 \\
\hline & Satisfied & 185.00 & 62.30 \\
\hline & Very satisfied & 22.00 & 7.40 \\
\hline \multirow{5}{*}{$\begin{array}{l}\text { Do you think home visiting physical therapy is appropriate for patients who } \\
\text { need long-term treatment? }\end{array}$} & Very dissatisfied & 1.00 & .30 \\
\hline & Dissatisfied & 15.00 & 5.10 \\
\hline & Neutral & 74.00 & 24.90 \\
\hline & Satisfied & 182.00 & 61.30 \\
\hline & Very satisfied & 25.00 & 8.40 \\
\hline \multirow[t]{5}{*}{ Do you think early discharge from hospital can help reduce hospital fees? } & Very dissatisfied & 4.00 & 1.30 \\
\hline & Dissatisfied & 30.00 & 10.10 \\
\hline & Neutral & 101.00 & 34.00 \\
\hline & Satisfied & 142.00 & 47.80 \\
\hline & Very satisfied & 20.00 & 6.70 \\
\hline \multirow{5}{*}{$\begin{array}{l}\text { Do you think that with home visiting physical therapy, intensive care is } \\
\text { possible and effective treatment can be provided? }\end{array}$} & Very dissatisfied & 1.00 & .30 \\
\hline & Dissatisfied & 18.00 & 6.10 \\
\hline & Neutral & 84.00 & 28.30 \\
\hline & Satisfied & 161.00 & 54.20 \\
\hline & Very satisfied & 33.00 & 11.10 \\
\hline \multirow{5}{*}{$\begin{array}{l}\text { Do you think with that home visiting physical therapy can provide treatment } \\
\text { without causing discomfort to patients in front of others? }\end{array}$} & Very dissatisfied & .00 & .00 \\
\hline & Dissatisfied & 12.00 & 4.00 \\
\hline & Neutral & 60.00 & 20.20 \\
\hline & Satisfied & 180.00 & 60.60 \\
\hline & Very satisfied & 45.00 & 15.20 \\
\hline \multirow{5}{*}{$\begin{array}{l}\text { Do you think that with home visiting physical therapy, it is easier for family } \\
\text { members to take care of patients? }\end{array}$} & Very dissatisfied & .00 & .00 \\
\hline & Dissatisfied & 20.00 & 6.70 \\
\hline & Neutral & 80.00 & 26.90 \\
\hline & Satisfied & 161.00 & 54.20 \\
\hline & Very satisfied & 36.00 & 12.20 \\
\hline \multirow{5}{*}{$\begin{array}{l}\text { Do you think that home visiting physical therapy can be provided at a time } \\
\text { patients choose by way of advance appointment? }\end{array}$} & Very dissatisfied & .00 & .00 \\
\hline & Dissatisfied & 11.00 & 3.70 \\
\hline & Neutral & 89.00 & 30.00 \\
\hline & Satisfied & 163.00 & 54.90 \\
\hline & Very satisfied & 34.00 & 11.40 \\
\hline
\end{tabular}

therapy can be provided at a time patients choose by way

of advance appointment?" ( $p<.05)$, (Table 4). 
Table 4. Need for home visiting physical therapy in terms of physical therapist characteristics.

\begin{tabular}{|c|c|c|c|c|c|}
\hline \multicolumn{3}{|c|}{ characteristics } & $\mathrm{N}$ & $\mathrm{M} \pm \mathrm{SD}$ & $\mathrm{t}$ or $\mathrm{F}$ \\
\hline \multirow{36}{*}{$\begin{array}{l}\text { Do you think home } \\
\text { visiting physical therapy } \\
\text { helps reduce the } \\
\text { inconvenience associated } \\
\text { with commuting to/from } \\
\text { hospital? }\end{array}$} & Sex & Male & 97.00 & $3.65 \pm .76$ & -1.47 \\
\hline & & Female & 200.00 & $3.77 \pm .61$ & \\
\hline & Age & $20 \sim 29$ years old & 116.00 & $3.72 \pm .60$ & .08 \\
\hline & & $30 \sim 39$ years old & 126.00 & $3.72 \pm .69$ & \\
\hline & & 40 years old $<$ & 55.00 & $3.76 \pm .74$ & \\
\hline & Education & College graduate & 177.00 & $3.70 \pm .73$ & .74 \\
\hline & & University graduate & 97.00 & $3.75 \pm .54$ & \\
\hline & & A postgraduate school & 23.00 & $3.87 \pm .63$ & \\
\hline & Marital status & Unmarried & 183.00 & $3.68 \pm .67$ & 1.76 \\
\hline & & Married & 114.00 & $3.82 \pm .65$ & \\
\hline & Dependent family & No & 171.00 & $3.71 \pm .65$ & .51 \\
\hline & & Yes & 126.00 & $3.75 \pm .69$ & \\
\hline & Monthly pay & $<1,500,000$ & 48.00 & $3.90 \pm .59$ & 1.65 \\
\hline & & $1,510,000 \sim 2,000,000$ & 130.00 & $3.75 \pm .61$ & \\
\hline & & $2,010,000 \sim 2,500,000$ & 57.00 & $3.68 \pm .65$ & \\
\hline & & $2,510,000 \sim 3,000,000$ & 42.00 & $3.64 \pm .79$ & \\
\hline & & $3,010,000<$ & 20.00 & $3.50 \pm .82$ & \\
\hline & Physical therapist career period & $<5$ years old & 113.00 & $3.78 \pm .62$ & 1.90 \\
\hline & & $6 \sim 9$ years old & 106.00 & $3.62 \pm .72$ & \\
\hline & & 10 years old $<$ & 78.00 & $3.57 \pm .87$ & \\
\hline & Position & Manager of physical therapy & 76.00 & $3.67 \pm .68$ & -.97 \\
\hline & & Physical therapy & 214.00 & $3.76 \pm .65$ & \\
\hline & Working week & $<40$ times & 66.00 & $3.77 \pm .57$ & .50 \\
\hline & & $41 \sim 49$ times & 208.00 & $3.71 \pm .70$ & \\
\hline & & 50 times $<$ & 23.00 & $3.83 \pm .49$ & \\
\hline & Site & Gwangju & 226.00 & $3.71 \pm .68$ & -.84 \\
\hline & & Jeonnam & 71.00 & $3.79 \pm .61$ & \\
\hline & Service period & $<5$ years old & 218.00 & $3.72 \pm .64$ & $3.96^{*}$ \\
\hline & & $6 \sim 9$ years old & 58.00 & $3.62 \pm .65$ & \\
\hline & & 10 years old $<$ & 21.00 & $3.90 \pm .67$ & \\
\hline & 1 day number of patient care & $<10$ peoples & 25.00 & $3.84 \pm .68$ & .22 \\
\hline & & $11 \sim 20$ peoples & 67.00 & $3.73 \pm .64$ & \\
\hline & & $21 \sim 30$ peoples & 71.00 & $3.73 \pm .67$ & \\
\hline & & $31 \sim 40$ peoples & 74.00 & $3.73 \pm .72$ & \\
\hline & & 41 peoples $<$ & 47.00 & $3.70 \pm .62$ & \\
\hline & & And so on & 13.00 & $3.62 \pm .50$ & \\
\hline
\end{tabular}




\begin{tabular}{|c|c|c|c|c|c|}
\hline \multirow{36}{*}{$\begin{array}{l}\text { Do you think home } \\
\text { visiting physical therapy } \\
\text { is appropriate for patients } \\
\text { who need long-term } \\
\text { treatment? }\end{array}$} & \multirow{2}{*}{ Sex } & Male & 97.00 & $3.67 \pm .76$ & \multirow[t]{2}{*}{-.92} \\
\hline & & Female & 200.00 & $3.75 \pm .67$ & \\
\hline & & $20 \sim 29$ years old & 116.00 & $3.68 \pm .71$ & \multirow[t]{3}{*}{.93} \\
\hline & & $30 \sim 39$ years old & 126.00 & $3.71 \pm .70$ & \\
\hline & & 40 years old $<$ & 55.00 & $3.84 \pm .69$ & \\
\hline & \multirow[t]{3}{*}{ Education } & College graduate & 177.00 & $3.70 \pm .70$ & \multirow[t]{3}{*}{.60} \\
\hline & & University graduate & 97.00 & $3.73 \pm .71$ & \\
\hline & & A postgraduate school & 23.00 & $3.87 \pm .69$ & \\
\hline & \multirow[t]{2}{*}{ Marital status } & Unmarried & 183.00 & $3.67 \pm .70$ & \multirow[t]{2}{*}{-1.79} \\
\hline & & Married & 114.00 & $3.82 \pm .70$ & \\
\hline & \multirow[t]{2}{*}{ Dependent family } & No & 171.00 & $3.66 \pm .70$ & \multirow[t]{2}{*}{-1.81} \\
\hline & & Yes & 126.00 & $3.81 \pm .70$ & \\
\hline & \multirow[t]{5}{*}{ Monthly pay } & $<1,500,000$ & 48.00 & $3.75 \pm .75$ & \multirow[t]{5}{*}{.04} \\
\hline & & $1,510,000 \sim 2,000,000$ & 130.00 & $3.72 \pm .72$ & \\
\hline & & $2,010,000 \sim 2,500,000$ & 57.00 & $3.72 \pm .55$ & \\
\hline & & $2,510,000 \sim 3,000,000$ & 42.00 & $3.69 \pm .71$ & \\
\hline & & $3,010,000<$ & 20.00 & $3.75 \pm .78$ & \\
\hline & \multirow[t]{3}{*}{ Physical therapist career period } & $<5$ years old & 113.00 & $3.76 \pm .68$ & \multirow[t]{3}{*}{1.24} \\
\hline & & $6 \sim 9$ years old & 106.00 & $3.60 \pm .75$ & \\
\hline & & 10 years old $<$ & 78.00 & $3.67 \pm .69$ & \\
\hline & \multirow[t]{2}{*}{ Position } & Manager of physical therapy & 76.00 & $3.67 \pm .68$ & \multirow[t]{2}{*}{-.78} \\
\hline & & Physical therapy & 214.00 & $3.74 \pm .69$ & \\
\hline & \multirow[t]{3}{*}{ Working week } & $<40$ times & 66.00 & $3.79 \pm .62$ & \multirow[t]{3}{*}{.86} \\
\hline & & $41 \sim 49$ times & 208.00 & $3.72 \pm .71$ & \\
\hline & & 50 times $<$ & 23.00 & $3.57 \pm .78$ & \\
\hline & \multirow[t]{2}{*}{ Site } & Gwangju & 226.00 & $3.73 \pm .70$ & \multirow[t]{2}{*}{.27} \\
\hline & & Jeonnam & 71.00 & $3.70 \pm .70$ & \\
\hline & \multirow[t]{3}{*}{ Service period } & $<5$ years old & 218.00 & $3.71 \pm .70$ & \multirow[t]{3}{*}{$5.87^{*}$} \\
\hline & & $6 \sim 9$ years old & 58.00 & $3.58 \pm .72$ & \\
\hline & & 10 years old $<$ & 21.00 & $3.94 \pm .61$ & \\
\hline & \multirow[t]{6}{*}{1 day number of patient care } & $<10$ peoples & 25.00 & $3.80 \pm .81$ & \multirow[t]{6}{*}{.24} \\
\hline & & $11 \sim 20$ peoples & 67.00 & $3.72 \pm .75$ & \\
\hline & & $21 \sim 30$ peoples & 71.00 & $3.76 \pm .62$ & \\
\hline & & $31 \sim 40$ peoples & 74.00 & $3.73 \pm .68$ & \\
\hline & & 41 peoples $<$ & 47.00 & $3.64 \pm .70$ & \\
\hline & & And so on & 13.00 & $3.69 \pm .75$ & \\
\hline
\end{tabular}




\begin{tabular}{|c|c|c|c|c|c|}
\hline \multirow{35}{*}{$\begin{array}{l}\text { Do you think early } \\
\text { discharge from hospital } \\
\text { can help reduce hospital } \\
\text { fees? }\end{array}$} & \multirow{2}{*}{ Sex } & Male & $\begin{array}{r}97.00 \\
20000\end{array}$ & $\begin{array}{r}3.54 \pm .87 \\
3.46+79\end{array}$ & .75 \\
\hline & & $\begin{array}{l}\text { Female } \\
20 \sim 29 \text { years old }\end{array}$ & $\begin{array}{l}200.00 \\
11600\end{array}$ & $\begin{array}{l}3.46 \pm .19 \\
3.43+82\end{array}$ & \\
\hline & & $30 \sim 39$ vears old & 12600 & $348 \pm 82$ & \multirow{2}{*}{.79} \\
\hline & & 40 years old $<$ & 55.00 & $3.60 \pm .83$ & \\
\hline & \multirow[t]{3}{*}{ Education } & College graduate & 177.00 & $3.46 \pm .78$ & \multirow[t]{3}{*}{2.79} \\
\hline & & University graduate & 97.00 & $3.44 \pm .88$ & \\
\hline & & A postgraduate school & 23.00 & $3.87 \pm .76$ & \\
\hline & \multirow[t]{2}{*}{ Marital status } & Unmarried & 183.00 & $3.40 \pm .81$ & \multirow[t]{2}{*}{$2.31^{*}$} \\
\hline & & Married & 114.00 & $3.62 \pm .81$ & \\
\hline & \multirow[t]{2}{*}{ Dependent family } & No & 171.00 & $3.83 \pm .81$ & \multirow[t]{2}{*}{$-2.59^{*}$} \\
\hline & & Yes & 126.00 & $3.63 \pm .81$ & \\
\hline & \multirow[t]{5}{*}{ Monthly pay } & $<1,500,000$ & 48.00 & $3.52 \pm .96$ & \multirow[t]{5}{*}{.44} \\
\hline & & $1,510,000 \sim 2,000,000$ & 130.00 & $3.47 \pm .74$ & \\
\hline & & $2,010,000 \sim 2,500,000$ & 57.00 & $3.56 \pm .78$ & \\
\hline & & $2,510,000 \sim 3,000,000$ & 42.00 & $3.36 \pm .87$ & \\
\hline & & $3,010,000<$ & 20.00 & $3.55 \pm .88$ & \\
\hline & \multirow{3}{*}{ Physical therapist career period } & $<5$ years old & 113.00 & $3.54 \pm .81$ & \multirow[t]{3}{*}{1.72} \\
\hline & & $6 \sim 9$ years old & 106.00 & $3.36 \pm .79$ & \\
\hline & & 10 years old $<$ & 78.00 & $3.29 \pm .96$ & \\
\hline & \multirow[t]{2}{*}{ Position } & Manager of physical therapy & 76.00 & $3.46 \pm .77$ & \multirow[t]{2}{*}{-.41} \\
\hline & & Physical therapy & 214.00 & $3.50 \pm .83$ & \\
\hline & \multirow[t]{3}{*}{ Working week } & $<40$ times & 66.00 & $3.52 \pm .70$ & \multirow[t]{3}{*}{.37} \\
\hline & & $41 \sim 49$ times & 208.00 & $3.49 \pm .84$ & \\
\hline & & 50 times $<$ & 23.00 & $3.35 \pm .88$ & \\
\hline & \multirow[t]{2}{*}{ Site } & Gwangju & 226.00 & $3.48 \pm .83$ & \multirow[t]{2}{*}{-.26} \\
\hline & & Jeonnam & 71.00 & $3.51 \pm .77$ & \\
\hline & \multirow[t]{3}{*}{ Service period } & $<5$ years old & 218.00 & $3.43 \pm .82$ & \multirow[t]{3}{*}{$3.55^{*}$} \\
\hline & & $6 \sim 9$ years old & 58.00 & $3.39 \pm .80$ & \\
\hline & & 10 years old $<$ & 21.00 & $3.69 \pm .81$ & \\
\hline & \multirow[t]{6}{*}{1 day number of patient care } & $<10$ peoples & 25.00 & $3.48 \pm .96$ & \multirow[t]{6}{*}{.43} \\
\hline & & $11 \sim 20$ peoples & 67.00 & $3.60 \pm .78$ & \\
\hline & & $21 \sim 30$ peoples & 71.00 & $3.49 \pm .75$ & \\
\hline & & $31 \sim 40$ peoples & 74.00 & $3.41 \pm .85$ & \\
\hline & & 41 peoples $<$ & 47.00 & $3.47 \pm .88$ & \\
\hline & & And so on & 13.00 & $3.38 \pm .65$ & \\
\hline
\end{tabular}




\begin{tabular}{|c|c|c|c|c|c|}
\hline \multirow{36}{*}{$\begin{array}{l}\text { Do you think that with } \\
\text { home visiting physical } \\
\text { therapy, intensive care } \\
\text { is possible and effective } \\
\text { treatment can be } \\
\text { provided? }\end{array}$} & \multirow[t]{2}{*}{ Sex } & Male & 97.00 & $3.73 \pm .85$ & \multirow[t]{2}{*}{.55} \\
\hline & & Female & 200.00 & $3.68 \pm .71$ & \\
\hline & \multirow[t]{3}{*}{ Age } & $20 \sim 29$ years old & 116.00 & $3.78 \pm .71$ & \multirow[t]{3}{*}{2.67} \\
\hline & & $30 \sim 39$ years old & 126.00 & $3.58 \pm .80$ & \\
\hline & & 40 years old $<$ & 55.00 & $3.80 \pm .73$ & \\
\hline & \multirow[t]{3}{*}{ Education } & College graduate & 177.00 & $3.72 \pm .72$ & \multirow[t]{3}{*}{1.36} \\
\hline & & University graduate & 97.00 & $3.61 \pm .82$ & \\
\hline & & A postgraduate school & 23.00 & $3.87 \pm .76$ & \\
\hline & \multirow[t]{2}{*}{ Marital status } & Unmarried & 183.00 & $3.69 \pm .73$ & \multirow[t]{2}{*}{-.08} \\
\hline & & Married & 114.00 & $3.70 \pm .81$ & \\
\hline & \multirow[t]{2}{*}{ Dependent family } & No & 171.00 & $3.64 \pm .75$ & \multirow[t]{2}{*}{-1.42} \\
\hline & & Yes & 126.00 & $3.77 \pm .77$ & \\
\hline & \multirow[t]{5}{*}{ Monthly pay } & $<1,500,000$ & 48.00 & $3.88 \pm .76$ & \multirow[t]{5}{*}{1.24} \\
\hline & & $1,510,000 \sim 2,000,000$ & 130.00 & $3.68 \pm .70$ & \\
\hline & & $2,010,000 \sim 2,500,000$ & 57.00 & $3.61 \pm .72$ & \\
\hline & & $2,510,000 \sim 3,000,000$ & 42.00 & $3.60 \pm .91$ & \\
\hline & & $3,010,000<$ & 20.00 & $3.85 \pm .81$ & \\
\hline & \multirow[t]{3}{*}{ Physical therapist career period } & $<5$ years old & 113.00 & $3.75 \pm .73$ & \multirow[t]{3}{*}{2.87} \\
\hline & & $6 \sim 9$ years old & 106.00 & $3.60 \pm .84$ & \\
\hline & & 10 years old $<$ & 78.00 & $3.38 \pm .80$ & \\
\hline & \multirow[t]{2}{*}{ Position } & Manager of physical therapy & 76.00 & $3.68 \pm .73$ & \multirow[t]{2}{*}{-.31} \\
\hline & & Physical therapy & 214.00 & $3.71 \pm .76$ & \\
\hline & \multirow[t]{3}{*}{ Working week } & $<40$ times & 66.00 & $3.68 \pm .66$ & \multirow[t]{3}{*}{1.59} \\
\hline & & $41 \sim 49$ times & 208.00 & $3.73 \pm .77$ & \\
\hline & & 50 times $<$ & 23.00 & $3.43 \pm .84$ & \\
\hline & \multirow[t]{2}{*}{ Site } & Gwangju & 226.00 & $3.69 \pm .76$ & \multirow[t]{2}{*}{-.27} \\
\hline & & Jeonnam & 71.00 & $3.72 \pm .76$ & \\
\hline & \multirow[t]{3}{*}{ Service period } & $<5$ years old & 218.00 & $3.75 \pm .72$ & \multirow[t]{3}{*}{$5.77^{*}$} \\
\hline & & $6 \sim 9$ years old & 58.00 & $3.51 \pm .78$ & \\
\hline & & 10 years old $<$ & 21.00 & $3.87 \pm .72$ & \\
\hline & \multirow[t]{6}{*}{1 day number of patient care } & $<10$ peoples & 25.00 & $3.68 \pm .90$ & \multirow[t]{6}{*}{.63} \\
\hline & & $11 \sim 20$ peoples & 67.00 & $3.64 \pm .77$ & \\
\hline & & $21 \sim 30$ peoples & 71.00 & $3.73 \pm .69$ & \\
\hline & & $31 \sim 40$ peoples & 74.00 & $3.74 \pm .82$ & \\
\hline & & 41 peoples $<$ & 47.00 & $3.74 \pm .67$ & \\
\hline & & And so on & 13.00 & $3.38 \pm .65$ & \\
\hline
\end{tabular}




\begin{tabular}{|c|c|c|c|c|c|}
\hline \multirow{36}{*}{$\begin{array}{l}\text { Do you think with that } \\
\text { home visiting physical } \\
\text { therapy can provide } \\
\text { treatment without causing } \\
\text { discomfort to patients in } \\
\text { front of others? }\end{array}$} & Sex & Male & 97.00 & $3.88 \pm .75$ & .13 \\
\hline & & Female & 200.00 & $3.87 \pm .68$ & \\
\hline & Age & $20 \sim 29$ years old & 116.00 & $3.92 \pm .67$ & 1.24 \\
\hline & & $30 \sim 39$ years old & 126.00 & $3.79 \pm .73$ & \\
\hline & & 40 years old $<$ & 55.00 & $3.93 \pm .72$ & \\
\hline & Education & College graduate & 177.00 & $3.86 \pm .66$ & .43 \\
\hline & & University graduate & 97.00 & $3.86 \pm .76$ & \\
\hline & & A postgraduate school & 23.00 & $4.00 \pm .80$ & \\
\hline & Marital status & Unmarried & 183.00 & $3.85 \pm .71$ & -.50 \\
\hline & & Married & 114.00 & $3.89 \pm .70$ & \\
\hline & Dependent family & No & 171.00 & $3.83 \pm .71$ & -1.08 \\
\hline & & Yes & 126.00 & $3.92 \pm .70$ & \\
\hline & Monthly pay & $<1,500,000$ & 48.00 & $3.98 \pm .75$ & .56 \\
\hline & & $1,510,000 \sim 2,000,000$ & 130.00 & $3.88 \pm .67$ & \\
\hline & & $2,010,000 \sim 2,500,000$ & 57.00 & $3.77 \pm .70$ & \\
\hline & & $2,510,000 \sim 3,000,000$ & 42.00 & $3.86 \pm .71$ & \\
\hline & & $3,010,000<$ & 20.00 & $3.85 \pm .81$ & \\
\hline & Physical therapist career period & $<5$ years old & 113.00 & $3.92 \pm .69$ & $5.83^{*}$ \\
\hline & & $6 \sim 9$ years old & 106.00 & $3.84 \pm .67$ & \\
\hline & & 10 years old $<$ & 78.00 & $3.38 \pm .80$ & \\
\hline & Position & Manager of physical therapy & 76.00 & $3.82 \pm .63$ & -.97 \\
\hline & & Physical therapy & 214.00 & $3.91 \pm .72$ & \\
\hline & Working week & $<40$ times & 66.00 & $3.71 \pm .71$ & 2.09 \\
\hline & & $41 \sim 49$ times & 208.00 & $3.91 \pm .69$ & \\
\hline & & 50 times $<$ & 23.00 & $3.91 \pm .73$ & \\
\hline & Site & Gwangju & 226.00 & $3.87 \pm .73$ & -.06 \\
\hline & & Jeonnam & 71.00 & $3.87 \pm .63$ & \\
\hline & Service period & $<5$ years old & 218.00 & $3.92 \pm .68$ & 2.61 \\
\hline & & $6 \sim 9$ years old & 58.00 & $3.75 \pm .71$ & \\
\hline & & 10 years old $<$ & 21.00 & $3.96 \pm .71$ & \\
\hline & 1 day number of patient care & $<10$ peoples & 25.00 & $3.88 \pm .66$ & .18 \\
\hline & & 11 20 peoples & 67.00 & $3.88 \pm .78$ & \\
\hline & & 21 30 peoples & 71.00 & $3.86 \pm .68$ & \\
\hline & & $31 \sim 40$ peoples & 74.00 & $3.88 \pm .70$ & \\
\hline & & 41 peoples $<$ & 47.00 & $3.89 \pm .66$ & \\
\hline & & And so on & 13.00 & $3.69 \pm .75$ & \\
\hline
\end{tabular}




\begin{tabular}{|c|c|c|c|c|c|}
\hline \multirow{36}{*}{$\begin{array}{l}\text { Do you think that with } \\
\text { home visiting physical } \\
\text { therapy, it is easier for } \\
\text { family members to take } \\
\text { care of patients? }\end{array}$} & \multirow[t]{2}{*}{ Sex } & Male & 97.00 & $3.70 \pm .79$ & \multirow[t]{2}{*}{-.25} \\
\hline & & Female & 200.00 & $3.73 \pm .75$ & \\
\hline & \multirow[t]{3}{*}{ Age } & $20 \sim 29$ years old & 116.00 & $3.75 \pm .71$ & \multirow[t]{3}{*}{.34} \\
\hline & & $30 \sim 39$ years old & 126.00 & $3.67 \pm .75$ & \\
\hline & & 40 years old $<$ & 55.00 & $3.75 \pm .91$ & \\
\hline & \multirow[t]{3}{*}{ Education } & College graduate & 177.00 & $3.65 \pm .78$ & \multirow[t]{3}{*}{$4.21^{*}$} \\
\hline & & University graduate & 97.00 & $3.74 \pm .75$ & \\
\hline & & A postgraduate school & 23.00 & $4.13 \pm .55$ & \\
\hline & \multirow[t]{2}{*}{ Marital status } & Unmarried & 183.00 & $3.70 \pm .74$ & \multirow[t]{2}{*}{-.51} \\
\hline & & Married & 114.00 & $3.75 \pm .81$ & \\
\hline & \multirow[t]{2}{*}{ Dependent family } & No & 171.00 & $3.67 \pm .74$ & \multirow[t]{2}{*}{-1.33} \\
\hline & & Yes & 126.00 & $3.79 \pm .79$ & \\
\hline & \multirow[t]{5}{*}{ Monthly pay } & $<1,500,000$ & 48.00 & $3.85 \pm .82$ & \multirow[t]{5}{*}{1.46} \\
\hline & & $1,510,000 \sim 2,000,000$ & 130.00 & $3.75 \pm .70$ & \\
\hline & & $2,010,000 \sim 2,500,000$ & 57.00 & $3.53 \pm .78$ & \\
\hline & & $2,510,000 \sim 3,000,000$ & 42.00 & $3.67 \pm .78$ & \\
\hline & & $3,010,000<$ & 20.00 & $3.80 \pm .83$ & \\
\hline & \multirow[t]{3}{*}{ Physical therapist career period } & $<5$ years old & 113.00 & $3.77 \pm .75$ & \multirow[t]{3}{*}{$3.44^{*}$} \\
\hline & & $6 \sim 9$ years old & 106.00 & $3.66 \pm .76$ & \\
\hline & & 10 years old $<$ & 78.00 & $3.33 \pm .86$ & \\
\hline & \multirow[t]{2}{*}{ Position } & Manager of physical therapy & 76.00 & $3.63 \pm .76$ & \multirow[t]{2}{*}{-1.34} \\
\hline & & Physical therapy & 214.00 & $3.77 \pm .75$ & \\
\hline & \multirow[t]{3}{*}{ Working week } & $<40$ times & 66.00 & $3.71 \pm .71$ & \multirow[t]{3}{*}{.51} \\
\hline & & $41 \sim 49$ times & 208.00 & $3.74 \pm .78$ & \\
\hline & & 50 times $<$ & 23.00 & $3.57 \pm .66$ & \\
\hline & \multirow[t]{2}{*}{ Site } & Gwangju & 226.00 & $3.68 \pm .78$ & \multirow[t]{2}{*}{-1.44} \\
\hline & & Jeonnam & 71.00 & $3.83 \pm .70$ & \\
\hline & \multirow[t]{3}{*}{ Service period } & $<5$ years old & 218.00 & $3.77 \pm .72$ & \multirow[t]{3}{*}{2.21} \\
\hline & & $6 \sim 9$ years old & 58.00 & $3.59 \pm .71$ & \\
\hline & & 10 years old $<$ & 21.00 & $3.81 \pm .86$ & \\
\hline & \multirow[t]{6}{*}{1 day number of patient care } & $<10$ peoples & 25.00 & $3.68 \pm .74$ & \multirow[t]{6}{*}{.22} \\
\hline & & $11 \sim 20$ peoples & 67.00 & $3.72 \pm .77$ & \\
\hline & & $21 \sim 30$ peoples & 71.00 & $3.70 \pm .80$ & \\
\hline & & $31 \sim 40$ peoples & 74.00 & $3.77 \pm .82$ & \\
\hline & & 41 peoples $<$ & 47.00 & $3.72 \pm .64$ & \\
\hline & & And so on & 13.00 & $3.54 \pm .66$ & \\
\hline
\end{tabular}




\begin{tabular}{|c|c|c|c|c|c|}
\hline \multirow{36}{*}{$\begin{array}{l}\text { Do you think that home } \\
\text { visiting physical therapy } \\
\text { can be provided at a } \\
\text { time patients choose by } \\
\text { way of advance } \\
\text { appointment? }\end{array}$} & Sex & Male & 97.00 & $3.78 \pm .72$ & .73 \\
\hline & & Female & 200.00 & $3.72 \pm .70$ & \\
\hline & Age & $20 \sim 29$ years old & 116.00 & $3.80 \pm .65$ & 2.17 \\
\hline & & $30 \sim 39$ years old & 126.00 & $3.64 \pm .72$ & \\
\hline & & 40 years old $<$ & 55.00 & $3.84 \pm .76$ & \\
\hline & Education & College graduate & 177.00 & $3.71 \pm .69$ & 2.33 \\
\hline & & University graduate & 97.00 & $3.72 \pm .73$ & \\
\hline & & A postgraduate school & 23.00 & $4.04 \pm .64$ & \\
\hline & Marital status & Unmarried & 183.00 & $3.72 \pm .70$ & .77 \\
\hline & & Married & 114.00 & $3.78 \pm .71$ & \\
\hline & Dependent family & No & 171.00 & $3.68 \pm .72$ & -1.61 \\
\hline & & Yes & 126.00 & $3.82 \pm .69$ & \\
\hline & Monthly pay & $<1,500,000$ & 48.00 & $3.83 \pm .75$ & .62 \\
\hline & & $1,510,000 \sim 2,000,000$ & 130.00 & $3.75 \pm .70$ & \\
\hline & & $2,010,000 \sim 2,500,000$ & 57.00 & $3.65 \pm .61$ & \\
\hline & & $2,510,000 \sim 3,000,000$ & 42.00 & $3.69 \pm .74$ & \\
\hline & & $3,010,000<$ & 20.00 & $3.85 \pm .74$ & \\
\hline & Physical therapist career period & $<5$ years old & 113.00 & $3.76 \pm .71$ & .66 \\
\hline & & $6 \sim 9$ years old & 106.00 & $3.74 \pm .69$ & \\
\hline & & 10 years old $<$ & 78.00 & $3.57 \pm .68$ & \\
\hline & Position & Manager of physical therapy & 76.00 & $3.70 \pm .69$ & -.90 \\
\hline & & Physical therapy & 214.00 & $3.78 \pm .69$ & \\
\hline & Working week & $<40$ times & 66.00 & $3.79 \pm .64$ & .26 \\
\hline & & $41 \sim 49$ times & 208.00 & $3.72 \pm .73$ & \\
\hline & & 50 times $<$ & 23.00 & $3.78 \pm .60$ & \\
\hline & Site & Gwangju & 226.00 & $3.73 \pm .71$ & -.65 \\
\hline & & Jeonnam & 71.00 & $3.79 \pm .67$ & \\
\hline & Service period & $<5$ years old & 218.00 & $3.79 \pm .66$ & $6.72 *$ \\
\hline & & $6 \sim 9$ years old & 58.00 & $3.56 \pm .73$ & \\
\hline & & 10 years old $<$ & 21.00 & $3.92 \pm .67$ & \\
\hline & 1 day number of patient care & $<10$ peoples & 25.00 & $3.68 \pm .74$ & .73 \\
\hline & & $11 \sim 20$ peoples & 67.00 & $3.87 \pm .67$ & \\
\hline & & $21 \sim 30$ peoples & 71.00 & $3.73 \pm .71$ & \\
\hline & & $31 \sim 40$ peoples & 74.00 & $3.74 \pm .74$ & \\
\hline & & 41 peoples $<$ & 47.00 & $3.64 \pm .64$ & \\
\hline & & And so on & 13.00 & $3.62 \pm .76$ & \\
\hline
\end{tabular}

${ }^{*} \mathrm{p}<.05$

$\mathrm{M} \pm \mathrm{SD}$ : mean \pm standard deviation 


\section{Discussion}

Home visiting physical therapy can enhance functions needed for daily home life and be effective on improving satisfaction of life and psychological stability (An, 2009; Jung et al., 2002). The therapy may have positive influence on motor skills and daily life behaviors of patients with stroke, and can solve problems of inconvenience associated with commuting to/from hospital to facilitate consistent treatment after discharge (Hwang et al., 2003; Yang, 1997). Thus, active participation from both physical therapists and patients is expected when home visiting physical therapy is implemented (Kim, 2011).

In this study, many of the physical therapists in Gwangju and Jeollanam-do knew the significance of home visiting physical therapy to some extent and thought it was necessary. When asked of what diseases required this type of therapy, they chose stroke followed by cerebral palsy and spinal cord injury, a selection that may be caused by the fact that physical therapists mainly see patients with such disorders. In addition, the therapists said that they mostly wanted to conduct physical therapy to activities of daily living treatment when they performed home visiting physical therapy, indicating that they focused on activities of daily living treatment in order to reduce inconvenience in home life after basic exercise and manual therapies were provided in hospitals. Many of the therapists argued that public institutions should be in charge of home visiting physical therapy, indicating that they wanted the government to approach and manage the therapy systematically and professionally. Meanwhile, the therapist thought that the time of visit should be decided after consultation with patients and one hour would be appropriate. As for the level of qualification for the physical therapist providing home visiting physical therapy, most of the therapist chose at least five years of clinical experience with appropriate education. The therapists mostly thought home visiting physical therapy was needed.
Almost half of them said that they knew of home visiting physical therapy to some extent, while the other half had just heard of it or did not know of it at all. The result may be because home visiting physical therapy has not been sufficiently promoted yet and the policies supporting the system are not complete. Systematic management and consistent implementation such as the development of various programs that are effective at improving functions of patients within their homes, increasing satisfaction with life, maintaining psychological stability, training of professional manpower, and development of evaluation methods and self exercise programs may be needed to implement home visiting physical therapy (Nho, 2007). In addition, it may be necessary to connect the system with local medical institutions and manpower expansion through specialization and classification of manpower on visiting medical services (Lee et al., 1996).

Home visiting physical therapy may greatly contribute to improving quality of life and providing sufficiently higher usefulness as it gains higher satisfaction from all ages, but the beneficiaries of the therapy was small enough that only $7.5 \%$ of patients received home visiting physical therapy for a year (Ahn and $\mathrm{Yu}, 2012$ ). The therapy is necessary both for physical therapist and for patient and is twice as economical as visiting medical institution in terms of cost and convenience, requiring the foundation on which the therapy could be expanded and activated as soon as possible (Kim, 2013).

Home visiting physical therapy is a necessary system to solve problems of inconvenience of physical therapist, patients, and caregivers. It urgently needs to be introduced and can provide patients with chronic disease with high-quality physical therapy service (Lee et al., 2013). For its implementation without delay, efforts by of physical therapist, physical therapy association, and the government are required. Home visiting physical therapy have therapeutic effects on movement, self management, home life, and interpersonal relationships by improving activity 
and social participation. Home visiting physical therapy shows usefulness in satisfaction and need, having great potential in medical services in the future. In particular, guidance of daily life as the most important therapeutic approach may enhance capability of daily life, expand opportunities to perform various social participations, and have positive influence on satisfaction and quality of life. In foreign countries where the system is already implemented well, 450 individuals considered to be elderly, at least 80 years old, showed a decline in falling and improvement in balance ability after home visiting physical therapy (Robertson et al., 2001; Rosie and Taylor, 2007). Meanwhile, 759 patients who were at least 45 years old showed highly positive reactions in cost reduction and efficiency of treatment when they received home visiting physical therapy (Gitlin et al., 1995; Thomas et al., 2005). In order to promote functions, thus, not only the existing traditional medical approach but also holistic approach according with realistic and practical demands should be significantly considered. It may be needed to develop services customized for individual patients, and it is urgent to activate home visiting physical therapy as soon as possible.

\section{Conclusion}

The purpose of this study was to investigate thoughts of physical therapists in Gwangju and Jeollanam-do on home visiting physical therapy. The results showed that they felt the therapy was necessary in various domains but almost half of them just heard of it or did not know it, indicating that promotion and policies on home visiting physical therapy is required as soon as possible.

\section{References}

Ahn CS, Yu WJ. A study of management and satisfaction for home visiting physical therapy. J Korean Soc Phys Med. 2012;7(3):241-50.

An DH. Effects on improvement of activities of daily living through short-term home visiting physical therapy. Phys Ther Kor. 2007;14(2):53-60.

Bae SS. A strategy of the home visiting physical therapy. J Kor Phys Ther. 2003;15(4):180-9.

Duncan P, Studenski S, Richards L, et al. Randomized clinical trial of therapeutic exercise in subacute stroke. Stroke. 2003;34(9):2173-80.

Gill TM, Baker DI, Gottschalk M, et al. A program to prevent functional decline in physically frail, elderly persons who live at home. N Engl J Med. 2002;347(14): 1068-74.

Gitlin LN, Corcoran M, Leinmiller-Eckhardt S. Understanding the family perspective: An ethnographic framework for providing occupational therapy in the home. Am J Occup Ther. 1995;49(8):802-9.

Han DW, Moon TH, Lee EM, et al. Development of the system for home visiting physical therapy. J Kor Phys Ther. 2005;17(1):1-26.

Hwang BY, Lee EJ, Han SD. Effects of home visiting physical therapy on motor function and $\mathrm{ADL}$ in the persons with chronic stroke. J Kor Phys Ther. 2003;15(4): 319-30.

Jung HS, Park HS, Park TS, et al. A survey of patient satisfaction with physical therapy service. Phys Ther Kor. 2002;9(1):97-110.

Kim GY. A study on the introduction of home-based physical therapy rehabilitation services. Doctor's Degree. Eulji University. 2013.

Kim JH, Lee KJ. Study on the awareness and the necessity of home and visiting physical therapy. J Korean Soc Phys Med. 2014;9(4):465-74.

Kim MS. The study of efficient way of home-based physical 
therapy. Master's Degree. Sahmyook University. 2011.

Kwon CS, Kim SY, Jang HJ. The effects of home visiting physical therapy on the motor function, activity of daily living, and pain for disabled veterans. J Korean Soc Phys Med. 2014;9(2):171-9.

Lee DJ, Lee YS, Jung SM, et al. The necessity and awareness of home visiting physical therapy in Gwang ju, Junnam area. J Korean Soc Integr Med. 2014;2(1):77-89.

Lee HS, Park DM, Kim CS. Home-visiting physical therapy. J Kor Phys Ther. 1996;8(1):91-8.

Lee MS, Kim MC, Kim GY. A study for home-based physical therapy service introduction through a group of professionals in-depth interview. J Korean Soc Phys Med. 2013;8(3):303-15.

Mangione KK, Lopopolo RB, Neff NP, et al. Interventions used by physical therapists in home care for people after hip fracture. Phys Ther. 2008;88(2):199-210.

Nho HY. A study on the improvement of the elderly medical insurance system in the aging society: Focused on the increase in medical expense for the aged. Master's Degree. Konyang University. 2007.

Robertson MC, Devlin N, Gardner MM, et al. Effectiveness and economic evaluation of a nurse delivered home exercise programme to prevent falls. 1: Randomized controlled trial. BMJ. 2001;322(3):697-702.

Rosie J, Taylor D. Sit-to-stand as home exercise for mobility-limited adults over 80 years of ageGrandstand system TM may keep you standing?. Age Ageing. 2007;36(5):555-62.

Thomas KS, Miller P, Doherty M, et al. Cost effectiveness of a two-year home exercise program for the treatment of knee pain. Arthritis Rheum. 2005;53(3):388-94.

Yang YA. A positive study on home-visiting physical therapy system. Master's Degree. Hanyang University. 1997.

Yoon TH. A study of the inclusion of home-based physical therapy service in the long term care insurance. Doctor's Degree. Hanyang University. 2009. 